CALT-68-2112

QUIC-97-030

quant-ph/9705031

\title{
Reliable Quantum Computers
}

\author{
John Preskill|' \\ California Institute of Technology, Pasadena, CA 91125, USA
}

\begin{abstract}
The new field of quantum error correction has developed spectacularly since its origin less than two years ago. Encoded quantum information can be protected from errors that arise due to uncontrolled interactions with the environment. Recovery from errors can work effectively even if occasional mistakes occur during the recovery procedure. Furthermore, encoded quantum information can be processed without serious propagation of errors. Hence, an arbitrarily long quantum computation can be performed reliably, provided that the average probability of error per quantum gate is less than a certain critical value, the accuracy threshold. A quantum computer storing about $10^{6}$ qubits, with a probability of error per quantum gate of order $10^{-6}$, would be a formidable factoring engine. Even a smaller, less accurate quantum computer would be able to perform many useful tasks.

This paper is based on a talk presented at the ITP Conference on Quantum Coherence and Decoherence, 15-18 December 1996.
\end{abstract}

\section{The golden age of quantum error correction}

Many of us are hopeful that quantum computers will become practical and useful computing devices some time during the 21st century. It is probably fair to say, though, that none of us can now envision exactly what the hardware of that machine of the future will be like; surely, it will be much different than the sort of hardware that experimental physicists are investigating these days. But of one thing we can be quite confident - that a practical quantum computer will incorporate some type of error correction into its operation. Quantum computers are far more susceptible to making errors than conventional digital computers, and some method of controlling and correcting those errors will be needed to prevent a quantum computer from crashing.

As recently as mid 1995, we did not have a clear idea how quantum error correction would work, or whether it would work. Indeed, there were a number of reasons for pessimism about whether quantum error correction could really be possible (Unruh 1995; Landauer 1995, 1996, 1997). First of all, although very sophisticated methods have been developed to correct errors in classical information (MacWilliams \& Sloane 1977), it is not immediately clear how to adapt these methods to correct the phase errors that plague quantum systems. Second, in a quantum computer, as in a classical analog computer, small errors can accumulate over time and eventually add up to large errors, and it is difficult to find methods that can prevent or correct such small errors. Third, to correct an error, we must first acquire some information about the nature of the error by making a measurement, and there is a danger that the measurement will destroy the delicate quantum information that is encoded in the device. Finally, to protect against errors we must encode information in a redundant manner. But a famous theorem (Wootters \& Zurek 1982; Dieks 1982) says that quantum information cannot be copied, so it is not obvious how to store information with the required redundancy.

But by now all of these apparent obstacles have been overcome - we now know that quantum error correction really is possible. The key conceptual point we have grasped is that we can fight

\footnotetext{
${ }^{1}$ preskill@theory.caltech.edu
} 
entanglement with entanglement. Entanglement can be our enemy, since entanglement of our device with the environment can conceal quantum information from us, and so cause errors. But entanglement can also be our friend - we can encode the information that we want to protect in entanglement, that is, in correlations involving a large number of qubits. This information, then, cannot be accessed if we measure just a few qubits. By the same token, though, the information cannot be damaged if the environment interacts with just a few qubits. Furthermore, we have learned that, although the quantum computer is in a sense an analog device, we can digitalize the errors that it makes. We deal with small errors by making appropriate measurements that project the state of our quantum computer onto either a state where no error has occurred, or a state with a large error, which can then be corrected with familiar methods. And we have seen that it is possible to measure the errors without measuring the data - we can acquire information about the precise nature of the error without acquiring any information about the quantum information encoded in our device (which would result in decoherence and failure of our computation). The central idea of quantum error correction is this: a small subspace of the Hilbert space of our device is designated as the code subspace. This space is carefully chosen so that all of the errors that we want to correct move the code space to mutually orthogonal error subspaces. We can make a measurement after our system has interacted with the environment that tells us in which of these mutually orthogonal subspaces the system resides, and hence infer exactly what type of error occurred. The error can then be repaired by applying an appropriate unitary transformation.

If we were pessimistic in 1995, there was good reason for optimism by late 1996. This past year has been a landmark year for quantum information; it is the year that we have learned how to resist and reverse the effects of decoherence. This discovery has important consequences for quantum computation, but it will also have broader ramifications. Here are some the milestones that have been reached this year: That quantum error correcting codes exist was first pointed out by Peter Shor (1995) and Andrew Steane (1996a) in the fall of '95. By early '96, Steane (1996b) and Calderbank and Shor (1996) had shown that good codes exist, that is, codes that are capable of correcting many errors. We learned from the work on random codes by Lloyd (1997) and by Bennett, DiVincenzo, Smolin, and Wootters (1996) that if we want to store quantum information for a while, then we can find a code that will enable us to recover the stored information with high fidelity if the probability of error per qubit is less than about $19 \%$.

But that $19 \%$ estimate of the allowable error rate is quite misleading, because if applies only under a very unrealistic assumption - that we can encode the information and perform the recovery flawlessly, without making any mistakes. In fact, encoding and recovery are themselves complex quantum computations, and errors will inevitably occur while we carry out these operations. Thus, we need to find methods for recovering from errors that are sufficiently robust that we can still recover the quantum information with high accuracy even when we make some errors during the recovery step. This is the problem of fault-tolerant recovery, and Peter Shor (1996) showed in a pioneering paper written last May that fault-tolerant recovery is possible if the error rate is not too high.? Of course, we want more than just to store quantum information; we want to be able to process the information and build up an interesting quantum computation. So we must show that it is possible to devise quantum gates that work efficiently on information that has been carefully encoded so as to be protected from errors. And in the same paper last May, Shor showed that fault-tolerant computation is indeed possible.

In August, Manny Knill and Raymond Laflamme (1996) showed that there is an accuracy threshold for storage of quantum information; that is, if the error rate is below a certain critical value, then it is possible to store an unknown quantum state with high fidelity for an indefinitely long time. In the Caltech group (Gottesman et al. 1996), we quickly recognized that it is possible to combine the ideas of Shor with those of Knill and Laflamme to show that there is also an accuracy threshold for computation; if the error rate is below a critical value, then it is possible to do an arbitrarily long quantum computation with a negligible probability of error. Similar conclusions were reported by

\footnotetext{
${ }^{2}$ Methods for fault-tolerant recovery were also developed independently by Alesha Kitaev (1996a).
} 

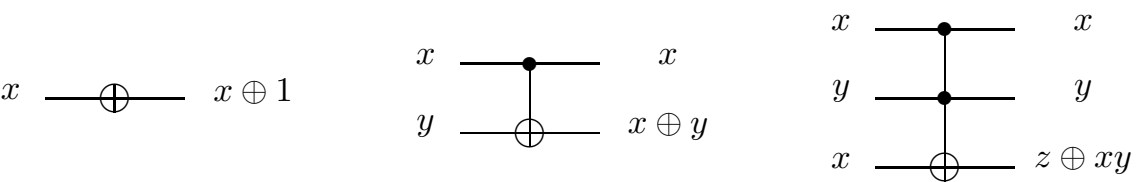

Figure 1: Diagrammatic notation for the NOT gate, the XOR (controlled-NOT) gate, and the Toffoli (controlled-controlled-NOT) gate.

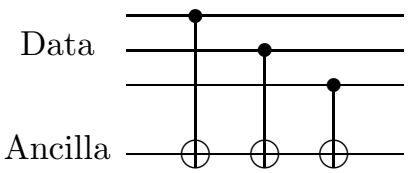

Bad!

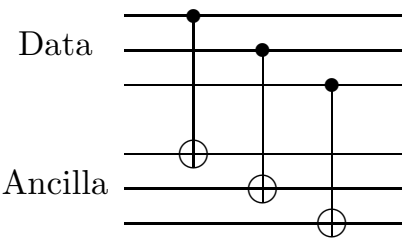

Good!

Figure 2: The first law. A bad circuit that uses the same ancilla bit several times, and a good circuit that uses each ancilla bit only once.

Knill, Laflamme, and Zurek (1996, 1997), by Aharonov and Ben-Or (1996), and by Kitaev (1996b).

Hence we are now in a position to sharpen the challenge to designers and builders of the quantum computers of the future. We can say just how well the hardware of that machine will have to work if it is to be used to perform interesting computations, where we might define interesting as competitive with what the best digital computers can do today. But first let's review the basic elements of fault-tolerant quantum computation.

\section{The laws of fault-tolerant computation}

To perform fault-tolerant computation, we must (1) ensure that recovery from errors is performed reliably, (2) be able to implement gates that can process encoded information, and (3) control propagation of errors. When we apply a gate to, say, a pair of qubits, and one of the qubits has an error, then the error will tend to spread to the other qubit. We need to be careful to contain the infection. The procedures that must be followed to implement fault-tolerant computation can be codified in what I will call "the laws of fault-tolerant computation." These can be distilled from Shor's pioneering paper (Shor 1996).

The first law is (1) Don't use the same bit twice 3 A bad network of XOR gates that breaks this commandment is shown in Fig. 2 (using the notation of Fig. 价. The bit that I have called the ancilla is the target of several successive gates. If there is a single error in this ancilla bit, this network may propagate that error to the several other bits that are the sources of the XOR gates; hence the infection spreads virulently. A peculiarly quantum-mechanical feature is that, while even a classical XOR gate will propagate bit flip errors from the source to the target, for quantum gates we must also worry about phase errors, and the phase errors propagate in the opposite direction, from the target to the source. So in quantum computation we need to be especially careful about propagation of errors. To follow this law, we may redesign the network as shown, expanding the ancilla to several bits so that no single bit is acted on more than once.

The second law concerns how recovery from errors must be effected. To recover, we copy some

\footnotetext{
${ }^{3}$ A less snappy but more judicious version of this law would be: Avoid using the same qubit too many times.
} 


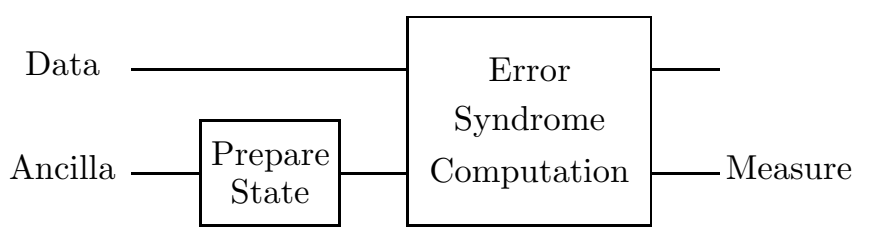

Figure 3: The second law. The ancilla must be prepared properly, so that the fault-tolerant syndrome measurement diagnoses the errors without spoiling the coherence of the data.

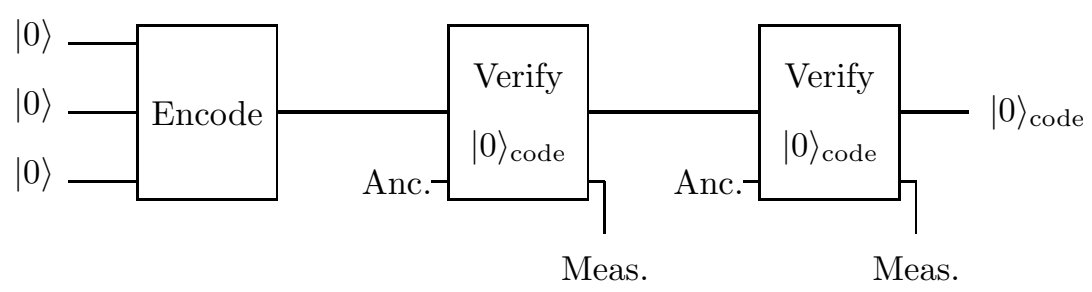

Figure 4: The third law. The encoder constructs $|0\rangle_{\text {code, }}$, and then a nondestructive measurement is performed (at least twice) to verify that the encoding was successful.

information from the data to an ancilla and then measure the ancilla to find an error syndrome that tells us what recovery operation is required. The second law says that we should (2) Copy the errors, not the data. If some of the encoded information is copied to the ancilla, the resulting entanglement of the data register and ancilla will cause decoherence and hence errors. To avoid this, we must prepare a special state of the ancilla before we copy any information, chosen so that by measuring the ancilla we acquire only information about the errors that have occurred, and learn nothing about the encoded data. (See Fig. 3.)

The third and fourth laws say that whenever we do anything, we should check (if possible) to make sure it has been done right. Often we will need to prepare a block that encodes a known quantum state, such as an encoded 0. The third law says that it pays to (3) Verify when you encode a known quantum state. During the encoding procedure we are quite vulnerable to errors - the power of the code to protect against errors is not yet in place, and a single error may propagate catastrophically. Therefore, we should carry out a measurement that checks that the encoding has been done correctly. Of course, the verification itself may be erroneous, so we must repeat the verification a few times before we have sufficient confidence that the encoding was correct.

That verification must be repeated is actually a special case of the fourth law, which says to (4) Repeat operations. An important application of this law is to the measurement of the syndrome that

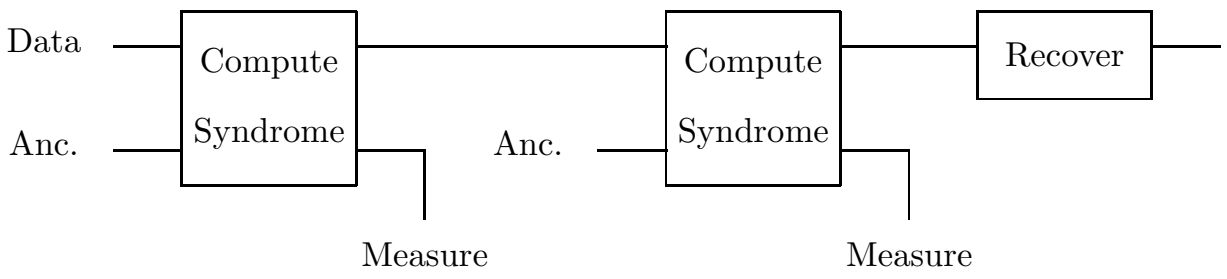

Figure 5: The fourth law. Operations such as syndrome measurement must be repeated to ensure accuracy. 


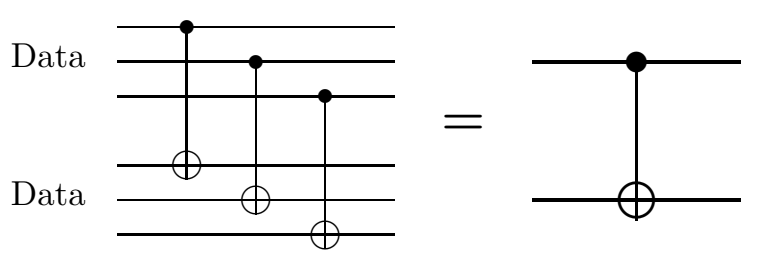

Figure 6: The fifth law. Transversal (bitwise) implementation of a fault-tolerant XOR gate.

precedes recovery. An error during syndrome measurement can both damage the data and result in an erroneous syndrome. If we mistakenly accept the measured syndrome and act accordingly, we will cause further damage instead of correcting the error. Therefore, it is important to be highly confident that the syndrome measurement is correct before we perform the recovery. To achieve sufficient confidence, we must repeat the syndrome measurement several times, in accord with the fourth law.

The fifth law is perhaps the most important and also the hardest to obey: (5) Use the right code. The code that we use for computation should have special properties so that we can apply quantum gates to the encoded information that operate efficiently and that adhere to the first four laws. For example, a good code for computation might be such that an XOR gate acting on encoded qubits is implemented as in Fig. 6-with a single XOR gate applied to each bit in both the source block and the target block. Then the gate acting on the encoded qubits obeys the first law and is fault-tolerant. f $^{-1}$

\section{Example: Steane's 7-qubit code}

To see how quantum error correction is possible, it is very instructive to study a particular code. A simple and important example of a quantum error-correcting code is the 7-qubit code devised by Andrew Steane (1996ab). This code enables us to store one qubit of quantum information (an arbitrary state in a two-dimensional Hilbert space) using altogether 7-qubits (by embedding the twodimensional Hilbert space in a space of dimension $2^{7}$ ). Steane's code is actually closely related to a familiar classical error-correcting code, the [7,4,3] Hamming code (MacWilliams \& Sloane 1977). To understand why Steane's code works, it is important to first understand the classical Hamming code.

The Hamming code uses a block of 7 bits to encode 4 bits of classical information; that is, there are $16=2^{4}$ strings of length 7 that are the valid codewords. The codewords can be characterized by a parity check matrix

$$
H=\left(\begin{array}{lllllll}
0 & 0 & 0 & 1 & 1 & 1 & 1 \\
0 & 1 & 1 & 0 & 0 & 1 & 1 \\
1 & 0 & 1 & 0 & 1 & 0 & 1
\end{array}\right)
$$

Each valid codeword is a 7-bit string $v_{\text {code }}$ that satisfies

$$
\sum_{k} H_{j k}\left(v_{\text {code }}\right)_{k}=0(\bmod 2)
$$

that is, the matrix $H$ annihilates each codeword in $\bmod 2$ arithmetic. Since $Z_{2}=\{0,1\}$ is a (finite) field, familiar results of linear algebra apply here. $H$ has three linearly independent rows and its kernel is spanned by four linearly independent column vectors. The 16 valid codewords are obtained by taking all possible linear combinations of these four strings, with coefficients chosen from $\{0,1\}$.

\footnotetext{
${ }^{4}$ When I gave the talk in December, it was not known how to implement fault-tolerant gates for most quantum codes. Since then Gottesman (1997a) has shown that fault-tolerant computation is possible for all the "stabilizer codes." Still, the fifth law is a good law; any code will do, but for some codes the fault-tolerant gates are less complicated.
} 
Now suppose that $v_{\text {code }}$ is an (unknown) valid codeword, and that a single (unknown) error occurs: one of the seven bits flips. We are assigned the task of determining which bit flipped, so that the error can be corrected. This trick can be performed by applying the parity check matrix to the string. Let $e_{i}$ denote the string with a one in the $i$ th place, and zeros elsewhere. Then when the $i$ th bit flips, $v_{\text {code }}$ becomes $v_{\text {code }}+e_{i}$. If we apply $H$ to this string we obtain

$$
H\left(v_{\text {code }}+e_{i}\right)=H e_{i}
$$

(because $H$ annihilates $v_{\text {code }}$ ), which is just the $i$ th column of the matrix $H$. Since all of the columns of $H$ are distinct, we can infer $i$; we have learned where the error occurred, and we can correct the error by flipping the $i$ th bit back. Thus, we can recover the encoded data unambiguously if only one bit flips; but if two or more different bits flip, the encoded data will be damaged. It is noteworthy that the quantity $\mathrm{He}_{i}$ reveals the location of the error without telling us anything about $v_{\text {code }}$; that is, without revealing the encoded information.

Steane's code generalizes this sort of classical error-correcting code to a quantum error-correcting code. The code uses a 7-qubit "block" to encode one qubit of quantum information, that is, we can encode an arbitrary state in a two-dimensional Hilbert space spanned by two states: the "logical zero" $|0\rangle_{\text {code }}$ and the "logical one" $|1\rangle_{\text {code. }}$. The code is designed to enable us to recover from an arbitrary error occurring in any of the 7 qubits in the block.

What do we mean by an arbitrary error? The qubit in question might undergo a random unitary transformation, or it might decohere by becoming entangled with states of the environment. Suppose that, if no error occurs, the qubit ought be in the state $a|0\rangle+b|1\rangle$. (Of course, this particular qubit might be entangled with others, so the coefficients $a$ and $b$ need not be complex numbers; they can be states that are orthogonal to both $|0\rangle$ and $|1\rangle$, which we assume are unaffected by the error.) Now if the qubit is afflicted by an arbitrary error, the resulting state can be expanded in the form:

$$
\begin{aligned}
& a|0\rangle+b|1\rangle \longrightarrow(a|0\rangle+b|1\rangle) \otimes\left|A_{\text {no error }} \quad\right\rangle_{\text {env }} \\
& +(a|1\rangle+b|0\rangle) \otimes\left|A_{\text {bit-flip }} \quad\right\rangle_{\text {env }} \\
& +(a|0\rangle-b|1\rangle) \otimes\left|A_{\text {phase-flip }}\right\rangle_{\text {env }} \\
& +(a|1\rangle-b|0\rangle) \otimes\left|A_{\text {both errors }}\right\rangle_{\text {env }},
\end{aligned}
$$

where each $|A\rangle_{\text {env }}$ denotes a state of the environment. We are making no particular assumption about the orthogonality or normalization of the $|A\rangle_{\mathrm{env}}$ states. 5 so Eq. [- entails no loss of generality. We conclude that the evolution of the qubit can be expressed as a linear combination of four possibilities: (1) no error occurs, (2) the bit flip $|0\rangle \leftrightarrow|1\rangle$ occurs, (3) the relative phase of $|0\rangle$ and $|1\rangle$ flips, (4) both a bit flip and a phase flip occur.

Now it is clear how a quantum error-correcting code should work (Steane 1996b; Knill \& Laflamme 1997). By making a suitable measurement, we wish to diagnose which of these four possibilities actually occurred. Of course, in general, the state of the qubit will be a linear combination of these four states, but the measurement should project the state onto the basis used in Eq. H. We can then proceed to correct the error by applying one of the four unitary transformations:

$$
\text { (1) } 1, \quad(2) X \equiv\left(\begin{array}{ll}
0 & 1 \\
1 & 0
\end{array}\right), \quad \text { (3) } Z \equiv\left(\begin{array}{cc}
1 & 0 \\
0 & -1
\end{array}\right), \quad \text { (4) } X \cdot Z,
$$

(and the measurement outcome will tell us which one to apply). By applying this transformation, we restore the qubit to its intended value, and completely disentangle the quantum state of the qubit from the state of the environment. It is essential, though, that in diagnosing the error, we learn nothing about the encoded quantum information, for to find out anything about the coefficients $a$ and $b$ in Eq. 1 would necessarily destroy the coherence of the qubit.

\footnotetext{
${ }^{5}$ Though, of course, the combined evolution of qubit plus environment is required to be unitary.
} 


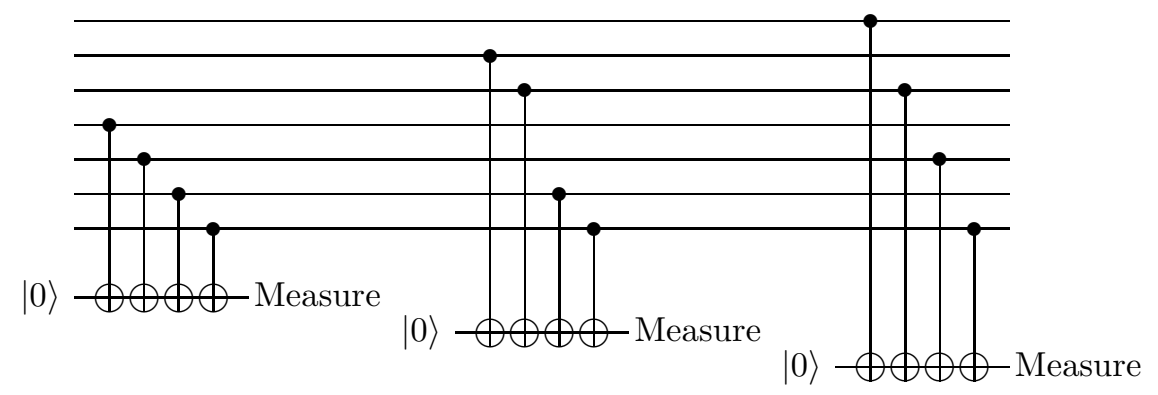

Figure 7: Computation of the bit-flip syndrome for Steane's 7-qubit code. Repeating the computation in the rotated basis diagnoses the phase-flip errors. To make the procedure fault tolerant, each ancilla qubit must be replaced by four qubits in a suitable state.

If we use Steane's code, a measurement meeting these criteria is possible. The logical zero is the equally weighted superposition of all of the even weight codewords of the Hamming code (those with an even number of 1's),

$$
\begin{aligned}
|0\rangle_{\text {code }}=\frac{1}{\sqrt{8}}\left(\sum_{\substack{\text { even } v \\
\text { Hamming }}}|v\rangle\right)=\frac{1}{\sqrt{8}} & (|0000000\rangle+|0001111\rangle+|0110011\rangle+|0111100\rangle \\
& +|1010101\rangle+|1011010\rangle+|1100110\rangle+|1101001\rangle),
\end{aligned}
$$

and the logical 1 is the equally weighted superposition of all of the odd weight codewords of the Hamming code (those with an odd number of 1's),

$$
\begin{aligned}
|1\rangle_{\text {code }}=\frac{1}{\sqrt{8}}\left(\sum_{\substack{\text { odd } v \\
\text { Hamming }}}|v\rangle\right)=\frac{1}{\sqrt{8}} & (|1111111\rangle+|1110000\rangle+|1001100\rangle+|1000011\rangle \\
& +|0101010\rangle+|0100101\rangle+|0011001\rangle+|0010110\rangle) .
\end{aligned}
$$

Since all of the states appearing in Eq. 6. and Eq. 77 are Hamming codewords, it is easy to detect a single bit flip in the block by doing a simple quantum computation, as illustrated in Fig. 7. We

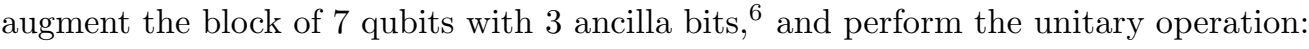

$$
|v\rangle \otimes|0\rangle_{\mathrm{anc}} \longrightarrow|v\rangle \otimes|H v\rangle_{\mathrm{anc}}
$$

where $H$ is the Hamming parity check matrix, and $|\cdot\rangle_{\text {anc }}$ denotes the state of the three ancilla bits. If we assume that only a single one of the 7 qubits in the block is in error, measuring the ancilla projects that qubit onto either a state with a bit flip or a state with no flip (rather than any nontrivial superposition of the two). If the bit does flip, the measurement outcome diagnoses which bit was affected, without revealing anything about the quantum information encoded in the block.

But to perform quantum error correction, we will need to diagnose phase errors as well as bit flip errors. To accomplish this, we observe (following Steane 1996ab) that we can change the basis for each qubit by applying the Hadamard rotation

$$
R=\frac{1}{\sqrt{2}}\left(\begin{array}{cc}
1 & 1 \\
1 & -1
\end{array}\right)
$$

Then phase errors in the $|0\rangle,|1\rangle$ basis become bit flip errors in the rotated basis

$$
|\tilde{0}\rangle \equiv \frac{1}{\sqrt{2}}(|0\rangle+|1\rangle), \quad|\tilde{1}\rangle \equiv \frac{1}{\sqrt{2}}(|0\rangle-|1\rangle) .
$$

\footnotetext{
${ }^{6}$ To make the procedure fault-tolerant, we will need to increase the number of ancilla bits as discussed in $\S 4$.
} 
It will therefore be sufficient if our code is able to diagnose bit flip errors in this rotated basis. But if we apply the Hadamard rotation to each of the 7 qubits, then Steane's logical 0 and logical 1 become in the rotated basis

$$
\begin{aligned}
& |\tilde{0}\rangle_{\text {code }}=\quad \frac{1}{4}\left(\sum_{\substack{\text { Hamming } \\
v \in}}|v\rangle\right)=\frac{1}{\sqrt{2}}\left(|0\rangle_{\text {code }}+|1\rangle_{\text {code }}\right) \\
& |\tilde{1}\rangle_{\text {code }}=\frac{1}{4}\left(\sum_{\substack{v \in \\
\text { Hamming }}}(-1)^{w t(v)}|v\rangle\right)=\frac{1}{\sqrt{2}}\left(|0\rangle_{\text {code }}-|1\rangle_{\text {code }}\right)
\end{aligned}
$$

(where $w t(v)$ denotes the weight of $v$ ). The key point is that $|\tilde{0}\rangle_{\text {code }}$ and $|\tilde{1}\rangle_{\text {code }}$, like $|0\rangle_{\text {code }}$ and $|1\rangle_{\text {code }}$, are superpositions of Hamming codewords. Hence, in the rotated basis, as in the original basis, we can perform the Hamming parity check to diagnose bit flips, which are phase flips in the original basis. Assuming that only one qubit is in error, performing the parity check in both bases completely diagnoses the error, and enables us to correct it.

In the above description of the error correction scheme, I assumed that the error affected only one of the qubits in the block. Clearly, this assumption as stated is not realistic; all of the qubits will typically become entangled with the environment to some degree. However, as we have seen, the procedure for determining the error syndrome will typically project each qubit onto a state in which no error has occurred. For each qubit, there is a non-zero probability of an error, assumed small, which we'll call $\epsilon$. Now we will make a very important assumption - that the errors acting on different qubits in the same block are completely uncorrelated with one another. Under this assumption, the probability of two errors is of order $\epsilon^{2}$, and so is much smaller than the probability of a single error if $\epsilon$ is small enough. So, to order $\epsilon$ accuracy, we can safely confine our attention to the case where at most one qubit per block is in error.

But in the (unlikely) event of two errors occurring in the same block of the code, our recovery procedure will typically fail. If two bits flip in the same block, then the Hamming parity check will misdiagnose the error. Recovery will restore the quantum state to the code subspace, but the encoded information in the block will undergo the bit flip

$$
|0\rangle_{\text {code }} \rightarrow|1\rangle_{\text {code }}, \quad|1\rangle_{\text {code }} \rightarrow|0\rangle_{\text {code }} .
$$

Similarly, if there are two phase errors in the same block, these are two bit flip errors in the rotated basis, so that after recovery the block will have undergone a bit flip in the rotated basis, or in the original basis the phase flip

$$
|0\rangle_{\text {code }} \rightarrow|0\rangle_{\text {code }}, \quad|1\rangle_{\text {code }} \rightarrow-|1\rangle_{\text {code }} .
$$

(If one qubit in the block has a phase error, and another one has a bit flip error, then recovery will be successful.)

Thus we have seen that Steane's code can enhance the reliability of stored quantum information. Suppose that we want to store one qubit in an unknown pure state $|\psi\rangle$. Due to imperfections in our storage device, the state $\rho_{\text {out }}$ that we recover will have suffered a loss of fidelity:

$$
F \equiv\left\langle\psi\left|\rho_{\text {out }}\right| \psi\right\rangle=1-\epsilon .
$$

But if we store the qubit using Steane's 7-qubit block code, if each of the 7-qubits is maintained with fidelity $F=1-\epsilon$, if the errors on the qubits are uncorrelated, and if we can perform error recovery, encoding, and decoding flawlessly (more on this below), then the encoded information can be maintained with an improved fidelity $F=1-O\left(\epsilon^{2}\right)$.

A qubit in an unknown state can be encoded using the circuit shown in Fig. 8. It is easiest to understand how the encoder works by using an alternative expression for the Hamming parity check matrix,

$$
H=\left(\begin{array}{lllllll}
1 & 0 & 0 & 1 & 0 & 1 & 1 \\
0 & 1 & 0 & 1 & 1 & 0 & 1 \\
0 & 0 & 1 & 1 & 1 & 1 & 0
\end{array}\right)
$$




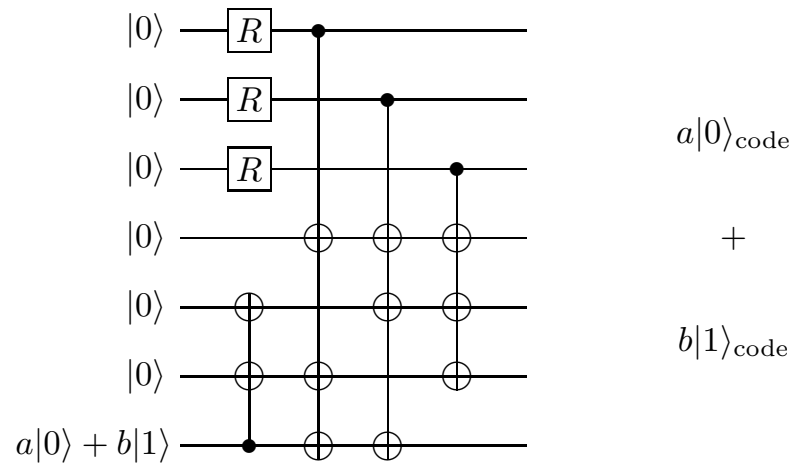

Figure 8: An encoding circuit for Steane's 7-qubit code.
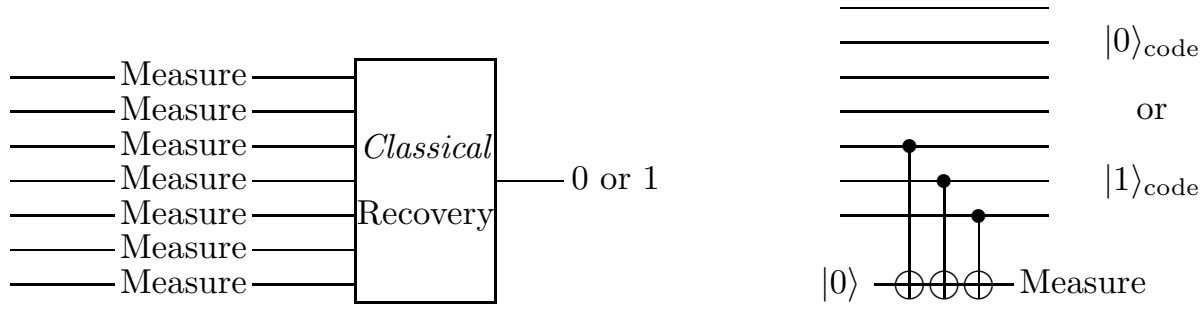

Figure 9: Destructive and nondestructive measurement of the logical qubit.

(This form of $H$ is obtained from the form in Eq. 1 by permuting the columns, which is just a relabeling of the bits in the block.) The even subcode of the Hamming code is actually the space spanned by the rows of $H$; so we see that (in this representation of $H$ ) the first three bits of the string completely characterize the data represented in the subcode. The remaining four bits are the parity bits that provide the redundancy needed to protect against errors. When encoding the unknown state $a|0\rangle+b|1\rangle$, the encoder first uses two XOR's to prepare the state $a|0000000\rangle+b|0000111\rangle$, a superposition of even and odd Hamming codewords. The rest of the circuit adds $|0\rangle_{\text {code }}$ to this state: the Hadamard $(R)$ rotations prepare an equally weighted superposition of all eight possible values for the first three bits in the block, and the remaining XOR gates switch on the parity bits dictated by $H$.

We will also want to be able to measure the encoded qubit, say by projecting onto the orthogonal basis $\left\{|0\rangle_{\text {code }},|1\rangle_{\text {code }}\right\}$. If we don't mind destroying the encoded block when we make the measurement, then it is sufficient to measure each of the seven qubits in the block by projecting onto the basis $\{|0\rangle,|1\rangle\}$; we then perform classical error correction on the measurement outcomes to obtain a Hamming codeword. The parity of that codeword is the value of the logical qubit. (The classical error correction step provides protection against measurement errors. For example, if the block is in the state $|0\rangle_{\text {code }}$, then two independent errors would have to occur in the measurement of the elementary qubits for the measurement of the logical qubit to yield the incorrect value $|1\rangle_{\text {code. }}$ )

In applications to quantum computation, we will need to perform a measurement that projects onto $\left\{|0\rangle_{\text {code }},|1\rangle_{\text {code }}\right\}$ without destroying the block. This task is accomplished by copying the parity of the block onto an ancilla qubit, and then measuring the ancilla. A circuit that performs a nondestructive measurement of the code block is shown in Fig. 9 .

Steane's 7-qubit code can recover from only a single error in the code block, but better codes can be constructed that can protect the information from up to $t$ errors within a single block, so that the 


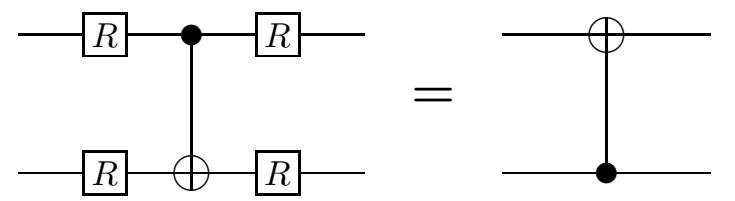

Figure 10: A useful identity. The source and the target of an XOR gate are interchanged if we perform a change of basis with Hadamard rotations.

encoded information can be maintained with a fidelity $F=1-O\left(\epsilon^{t+1}\right)$ (Steane 1996b; Calderbank and Shor 1996; Gottesman 1996; Calderbank et al. 1996, 1997). The current status of quantum coding theory is reviewed by Shor in these proceedings (Shor 1997).

\section{Fault-tolerant recovery}

But, of course, error recovery will never be flawless. Recovery is itself a quantum computation that will be prone to error. We must take care to design a recovery procedure for Steane's code that ensures that the probability of failure is of order $\epsilon^{2}$ (or of order $\epsilon^{t+1}$ for a code that corrects $t$ errors). Most critically, we must control propagation of error during the recovery procedure.

The circuit shown in Fig. 7 is not fault tolerant; it violates the first law. The XOR gates can propagate a single phase error in one of the ancilla bits to two different qubits in the data block, resulting in a phase error in the block that can occur with probability of order $\epsilon$. To adhere to the first law, we must expand each ancilla bit to four distinct bits, each of which is the target of just a single XOR gate. But now we must respect the second law: we must entangle the ancilla with the errors in the data block, but not with the quantum information encoded there, for entanglement of the ancilla with the encoded data will destroy the coherence of the data.

To meet this criterion, we prepare a Shor state of the ancilla before proceeding with the syndrome computation (Shor 1996). This is a state of four ancilla bits that is an equally weighted superposition of all even weight strings:

$$
\mid \text { Shor }\rangle_{\mathrm{anc}}=\frac{1}{\sqrt{8}} \sum_{\text {even } v}|v\rangle_{\mathrm{anc}} .
$$

To compute each bit of the syndrome, we prepare a Shor state, perform four XOR gates (with appropriate qubits in the data block as the sources and the four bits of the Shor state as the targets), and then measure the ancilla state. The syndrome bit is inferred from the parity of the four measured ancilla bits. The Shor state has been chosen so that only this parity can be inferred from the state of the ancilla - no other information about the data block gets imprinted there.

There are altogether 6 syndrome bits (3 to diagnose bit-flip errors and 3 to diagnose phase-flip errors), so the syndrome measurement uses 24 ancilla bits prepared in 6 Shor states, and 24 XOR gates.

(One way to obtain the phase-flip syndrome would be to first apply 7 parallel $R$ gates to the data block to rotate the basis, then to apply the XOR gates as in Fig. If (but with the ancilla expanded into a Shor state), and finally to apply $7 R$ gates to rotate the data back. However, we can use the identity represented in Fig. 10 to improve this procedure. By reversing the direction of the XOR gates (that is, by using the ancilla as the source and the data as the target), we can avoid applying the $R$ gates to the data, and hence can reduce the likelihood of damaging the data with faulty gates (Zalka 1996; Steane 1997).) 


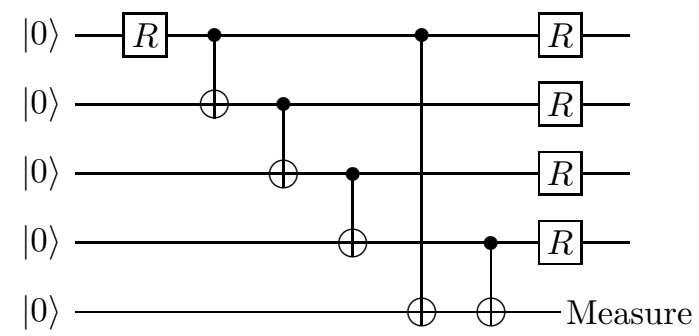

Figure 11: Construction and verification of the Shor state. If the measurement outcome is 1 , then the state is discarded and a new Shor state is prepared.

Due to error propagation, a single error that occurs during the preparation of the Shor state can result in two phase errors in the state, and these can both propagate to the data if the faulty ancilla is used for syndrome measurement. Therefore the Shor state must be tested for multiple phase errors before it is used (an instance of the third law), as in Fig. 11. If it fails the test, it should be discarded, and a new Shor state should be constructed.

Finally, a single error during syndrome measurement can result in a faulty syndrome. Thus, the syndrome measurement should be repeated to verify accuracy (the fourth law). When the same result is obtained twice in a row, the result can be safely accepted and recovery can proceed.

If we take all the precautions described above, then recovery will fail only if two independent errors occur, so the probability of an error occurring in the encoded block will be of order $\epsilon^{2}$. The error-correction procedure is fault-tolerant.

A somewhat streamlined fault-tolerant syndrome measurement procedure was suggested by Steane (1997). For the measurement of the bit-flip syndrome, we prepare the ancilla in the 7-qubit Steane state

$$
\mid \text { Steane }\rangle_{\text {anc }}=\frac{1}{4} \sum_{v \in \text { Hamming }}|v\rangle .
$$

(The Steane state may be prepared by applying the bitwise Hadamard rotation to the state $|0\rangle_{\text {code.) }}$ We may then XOR each qubit of the data block into the corresponding qubit of the ancilla, and measure the ancilla. Applying the Hamming parity-check matrix $H$ to the classical measurement outcome, we obtain the bit-flip syndrome. (Note the adherence to the second law: the procedure "copies" the data onto the ancilla, but the state of the ancilla has been carefully chosen to ensure that only the information about the error can be read by measuring the ancilla.) The same procedure is carried out in the rotated basis to find the phase-flip syndrome. The Steane procedure has the advantage over the Shor procedure that only 14 ancilla bits and 14 XOR gates are needed. But it also has the disadvantage that the ancilla preparation is more complex, so that the ancilla is somewhat more prone to error.

What about measurement and encoding? We have already noted that destructive measurement of the code block will be reliable if only one qubit in the block is in error. The nondestructive measurement depicted in Fig. 9 also need not be modified. Though the ancilla is the target of three successive XOR gates, phase errors feeding back into the block are not harmful because they cannot

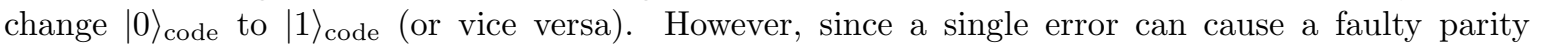
measurement, the measurement must be repeated (after error correction) to ensure accuracy to order $\epsilon^{2}$ (an instance of the fourth law).

In applications to quantum computation, we will need to repeatedly prepare the encoded state

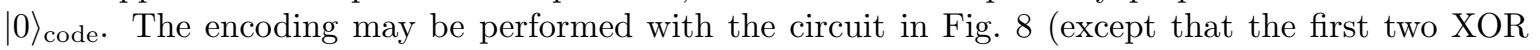
gates may be eliminated). However errors can propagate during the encoding procedure, so that a 
single error may suffice to cause an error in the encoded data. Hence it is important to verify the encoding, as stated in the third law, by performing nondestructive measurement of the block. In fact, the encoding step can actually be dispensed with. Whatever the initial state of the block, faulttolerant error correction will project it onto the space spanned by $\left\{|0\rangle_{\text {code }},|1\rangle_{\text {code }}\right\}$, and (verified) measurement will project out either $|0\rangle_{\text {code }}$ or $|1\rangle_{\text {code. }}$. If the result $|1\rangle_{\text {code }}$ is obtained, then the (bitwise) NOT operator can be applied to flip the block to the desired state $|0\rangle_{\text {code }}$.

If we wish to encode an unknown quantum state, then we use the encoding circuit in Fig. 8. Again, because of error propagation, a single error during encoding may cause an encoding failure. In this case, since no measurement can verify the encoding, the fidelity of the encoded state will inevitably be $F=1-O(\epsilon)$. However, encoding may still be worthwhile, since it may enable us to preserve the state with a reasonable fidelity for a longer time than if the state had remained unencoded.

Both Shor's and Steane's scheme for fault-tolerant syndrome measurement have been described here only for the 7-qubit code, but they can be adapted to more complex codes that have the capability to recover from many errors (DiVincenzo \& Shor 1996; Steane 1997). As the complexity of the code increases, Steane's scheme becomes substantially more efficient than Shor's.

\section{$5 \quad$ Fault-tolerant quantum gates}

We have seen that coding can protect quantum information. But we want to do more than store quantum information with high fidelity; we want to operate a quantum computer that processes the information. Of course, we could decode, perform a gate, and then re-encode, but that procedure would temporarily expose the quantum information to harm. Instead, if we want our quantum computer to operate reliably, we must be able to apply quantum gates directly to the encoded data, and these gates must respect the first law of fault tolerance if catastrophic propagation of error is to be avoided.

In fact, with Steane's 7-qubit code, there are a number of gates that can be easily implemented. Three single-qubit gates can all be applied bitwise; that is applying these gates to each of the 7 qubits in the block implements the same gate acting on the encoded qubit. We have already seen in Eq. 11 that the Hadamard rotation $R$ acts this way. The same is true for the NOT gate (since each odd parity Hamming codeword is the complement of an even parity Hamming codeword) 7 , and the phase gate

$$
P=\left(\begin{array}{cc}
1 & 0 \\
0 & i
\end{array}\right)
$$

(the odd Hamming codewords have weight $\equiv 3(\bmod 4)$ and the even codewords have weight $\equiv 0$ $(\bmod 4)$, so we actually apply $P^{-1}$ bitwise to implement $P$ ). The XOR gate can also be implemented bitwise; that is, by XOR'ing each bit of the source block into the corresponding bit of the target block. This works because the even codewords form a subcode, while the odd codewords are its nontrivial coset.

Thus there are simple fault-tolerant procedures for implementing the gates NOT, $R, P$, and XOR. But unfortunately, these gates do not by themselves form a universal set. To be able to perform arbitrary unitary transformations on encoded data, we will need to make a suitable addition to this set. Following, Shor (1996), we will add the 3 -qubit Toffoli gate, which is implemented by the procedure shown in Fig. $\left.12\right|^{8}$

Briefly, the procedure works as follows. First, three encoded ancilla blocks are prepared in a state of the form

$$
|A\rangle_{\mathrm{anc}} \equiv \sum_{a=0,1} \sum_{b=0,1}|a, b, a b\rangle_{\mathrm{anc}} .
$$

\footnotetext{
${ }^{7}$ Actually, we can implement the NOT acting on the encoded qubit with just 3 NOT's applied to selected qubits in the block.

${ }^{8}$ Knill et al. $(1996,1997)$ describe an alternative way of completing the universal set of gates.
} 


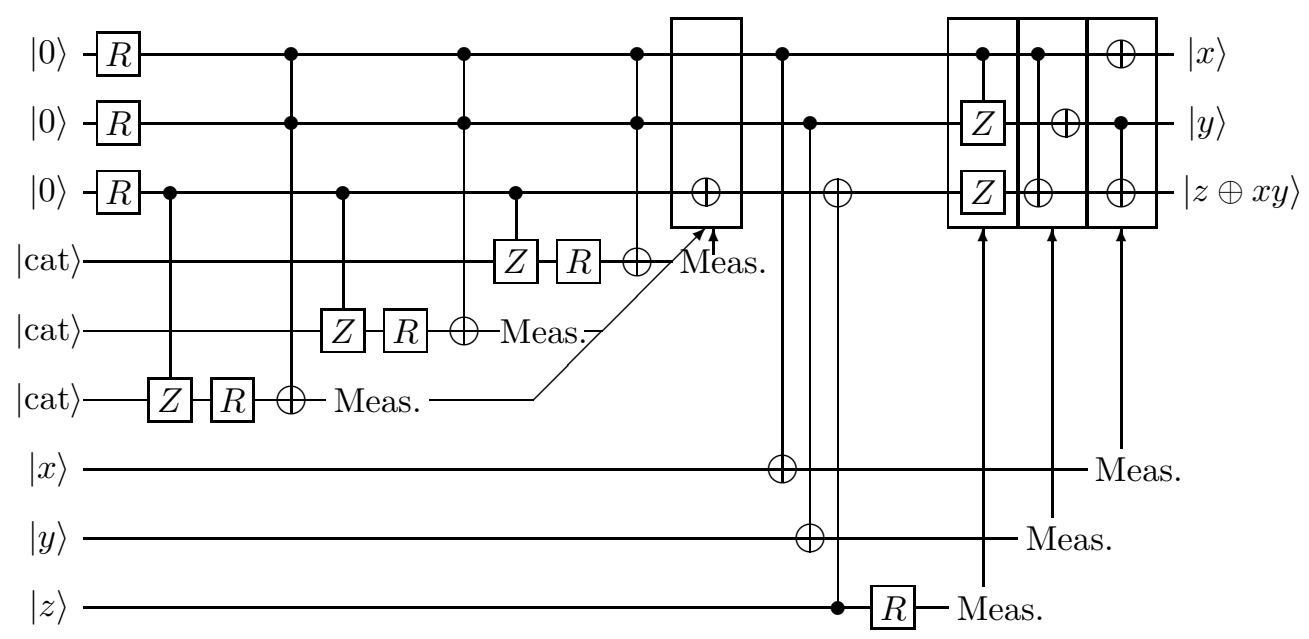

Figure 12: The fault-tolerant Toffoli gate. Each line represents a block of 7 qubits, and the gates are implemented transversally. For each measurement, the arrow points to the set of gates that is to be applied if the measurement outcome is 1 ; no action is taken if the outcome is 0 .

This preparation of the ancilla is performed by using a (verified) 7-bit "cat state"

$$
\mid \text { cat }\rangle=\frac{1}{\sqrt{2}}(|0000000\rangle+|1111111\rangle) \text {. }
$$

A few gates are performed, including a bitwise Toffoli gate with two ancilla blocks as the controls and the cat state as the target. Then the cat state is measured. (The measurement is repeated to ensure accuracy.) If the parity of the measurement outcomes is even, then the desired ancilla state $|A\rangle_{\text {anc }}$ has been successfully prepared; if the parity is odd, then the state $|A\rangle_{\text {anc }}$ can be obtained by applying a NOT to the third ancilla block.

Meanwhile, three data blocks have been waiting patiently for the ancilla to be ready. By applying three XOR gates and a Hadamard rotation, the state of the data and ancilla is transformed as

$$
\sum_{a=0,1} \sum_{b=0,1}|a, b, a b\rangle_{\mathrm{anc}}|x, y, z\rangle_{\mathrm{data}} \longrightarrow \sum_{a=0,1} \sum_{b=0,1} \sum_{w=0,1}(-1)^{w z}|a, b, a b \oplus z\rangle_{\mathrm{anc}}|x \oplus a, y \oplus b, w\rangle_{\mathrm{data}} .
$$

Now each data block is measured. If the measurement outcome is 0 , no action is taken, but if the measurement outcome is 1, then a particular set of gates is applied to the ancilla, as shown in Fig. 12, to complete the implementation of the Toffoli gate. Note that the original data blocks are destroyed by the procedure, and that what were initially the ancilla blocks become the new data blocks. The important thing about this construction is that all of the steps have been carefully designed to adhere to the laws of fault tolerance.

That the fault-tolerant gates form a discrete set is a bit of a nuisance, but it is also an unavoidable feature of any fault-tolerant scheme. It would not make sense for the fault-tolerant gates to form a continuum, for then how could we possibly avoid making an error by applying the wrong gate, a gate that differs from the intended one by a small amount? Anyway, since our fault-tolerant gates form a universal set, they suffice for approximating any desired unitary transformation to any desired accuracy.

Shor described how to generalize this fault tolerant set of gates to more complex codes that can correct more errors, and Gottesman (1997ab) has described an even more general procedure that can be applied to any of the known quantum codes. Thus, almost any quantum error-correcting code can 
be used for fault-tolerant computation. What then is the meaning of the fifth law? While any code can be used in principle, some codes are better than others. For example, there is a 5 -qubit code that can recover from one error (Bennett et al. 1996, Laflamme et al. 1996), and Gottesman has exhibited a universal set of fault-tolerant gates for this code. But the gate implementation is quite complex. The 7-qubit Steane code requires a larger block, but it is much more convenient for computation; the fifth law dictates that the Steane code should be preferred.

\section{The accuracy threshold for quantum computation}

Quantum error-correcting codes exist that can correct $t$ errors, where $t$ can be arbitrarily large. If we use such a code and we follow the laws of fault-tolerance, then an uncorrectable error will occur only if at least $t+1$ independent errors occur in a single block before recovery is completed. So if the probability of an error occurring per quantum gate, or the probability of a storage error occurring per unit of time, is of order $\epsilon$, then the probability of an error per gate acting on encoded data will be of order $\epsilon^{t+1}$, which is much smaller if $\epsilon$ is small enough. Indeed, it may seem that by choosing a code with $t$ as large as we please we can make the probability of error per gate as small as we please, but this turns out not to be the case, at least not for most codes. The trouble is that as we increase $t$, the complexity of the code increases sharply, and the complexity of the recovery procedure correspondingly increases. Eventually we reach the point where it takes so long to perform recovery that it is likely that $t+1$ errors will accumulate in a block before we can complete the recovery step, and the ability of the code to correct errors is thus compromised.

Suppose that the number of computational steps needed to perform the syndrome measurement increases with $t$ like a power $t^{b}$. Then the probability that $t+1$ errors accumulate before the measurement is complete will behave like

$$
\text { Block Error Probability } \sim\left(t^{b} \epsilon\right)^{t+1},
$$

where $\epsilon$ is the probability of error per step. We may then choose $t$ to minimize the error probability $\left(t \sim e^{-1} \epsilon^{-1 / b}\right.$, assuming $t$ is large), obtaining

$$
\text { Minimum Block Error Probability } \sim \exp \left(-e^{-1} b \epsilon^{-1 / b}\right) .
$$

Thus if we hope to carry out altogether $T$ cycles of error correction without any error occurring, then our gates must have an accuracy

$$
\epsilon \sim(\log T)^{-b} .
$$

Similarly, if we hope to perform a quantum computation with altogether $T$ quantum gates, elementary gates of this prescribed accuracy are needed.

In the procedure originally described by Shor (1996), the power characterizing the complexity of the syndrome measurement is $b=4$, and modest improvements $(b \sim 3)$ can be achieved with a better optimized procedure. The block size of the code used grows with $t$ like $t^{2}$, so when the code is chosen to optimize the error probability, the block size is of order $(\log T)^{2}$. Certainly, the scaling described by Eq. 24 is much more favorable than the accuracy $\epsilon \sim T^{-1}$ that would be required were coding not used at all. But for any given accuracy, there is a limit to how long a computation can proceed until errors become likely.

This limitation can be overcome by using a special kind of code, a concatenated code (Knill \& Laflamme 1996; Knill et al. 1996, 1997; Aharonov \& Ben-Or 1996; Kitaev 1996ab). To understand the concept of a concatenated code, imagine that we are using Steane's quantum error-correcting code that encodes a single qubit as a block of 7 qubits. But if we look more closely at one of the 7 qubits in the block with better resolution, we discover that it is not really a single qubit, but another block of 7 encoded using the same Steane code as before. And when we examine one of the 7 qubits in 


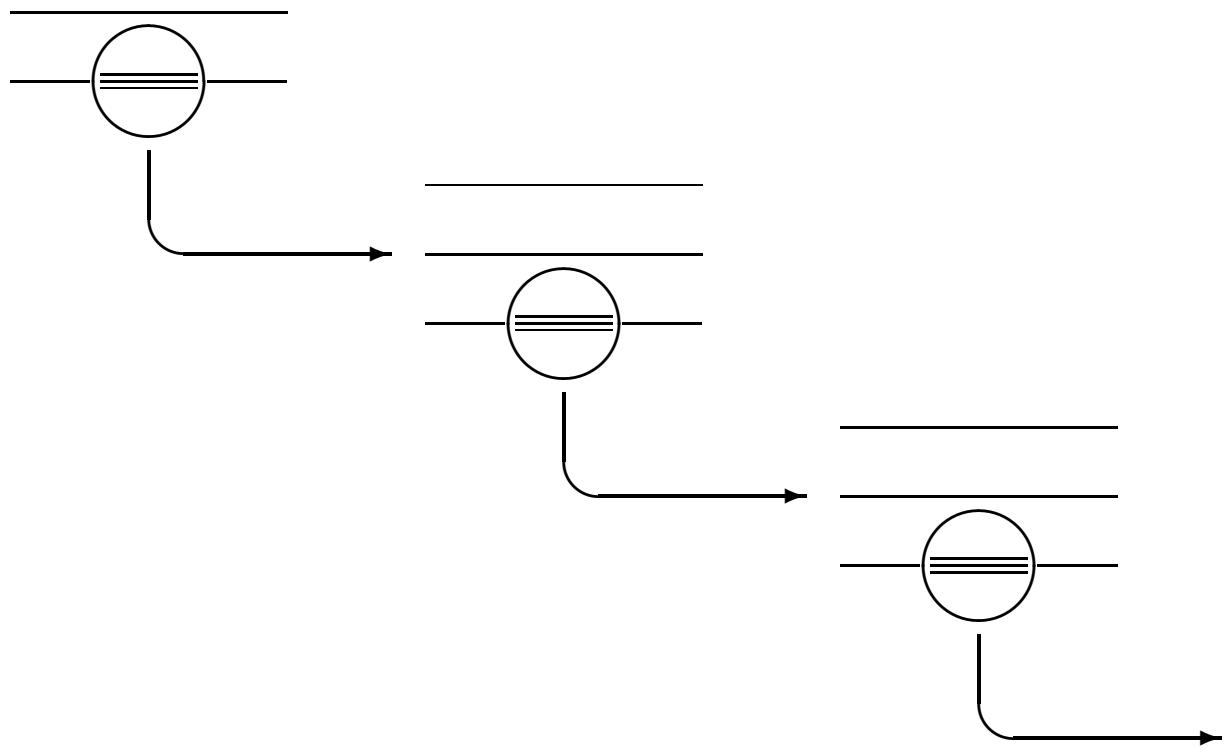

Figure 13: Concatenated coding. Each qubit in the block, when inspected at higher resolution, is itself an encoded subblock.

this block with higher resolution, we discover that it too is really a block of 7 qubits. And so on. (See Fig. 13.) If there are all together $L$ levels to this hierarchy of concatenation, then a single qubit is actually encoded in a block of size $7^{L}$. The reason that concatenation is useful is that it enables us to recover from errors more efficiently, by dividing and conquering. That is, we perform recovery most often at the lowest level of the hierarchy, on a block of size 7 , less often at the next highest level, on a block of size $7^{2}=49$, still less often at the next level, on a block of size $7^{3}=343$, and so on. With this method, the complexity of error correction does not grow so sharply as we increase the error-correcting capacity of our quantum code.

We have seen that Steane's 7-qubit code can recover from one error. If the probability of error per qubit is $\epsilon$, the errors are uncorrelated, and recovery is fault-tolerant, then the probability of a recovery failure is of order $\epsilon^{2}$. If we concatenate the code to construct a block of size $7^{2}$, then an error occurs in the block only if two of the subblocks of size 7 fail, which occurs with a probability of order $\left(\epsilon^{2}\right)^{2}$. And if we concatenate again, then an error occurs only if two subblocks of size $7^{2}$ fail, which occurs with a probability of order $\left(\left(\epsilon^{2}\right)^{2}\right)^{2}$. If there are all together $L$ levels of concatenation, then the probability of an error is or order $(\epsilon)^{2^{L}}$, while the block size is $7^{L}$. Now, if the error rate for our fundamental gates is small enough, then we can improve the probability of an error per gate by concatenating the code. If so, we can improve the performance even more by adding another level of concatenation. And so on. This is the origin of the accuracy threshold for quantum computation: if coding reduces the probability of error significantly, then we can make the error rate arbitrarily small by adding enough levels of concatenation. But if the error rates are too high to begin with, then coding will make things worse instead of better.

To analyze this situation, we must first adopt a particular model of the errors, and I will choose the simplest possible quasi-realistic model: uncorrelated stochastic errors. 9 In each computational time step, each qubit in the device becomes entangled with the environment as in Eq. A, but where we assume that the four states of the environment are mutually orthogonal, and that the three "error states" have equal norms. Thus the three types of errors (bit flip, phase flip, both) are assumed to be equally likely. The total probability of error in each time step is denoted $\epsilon_{\text {store }}$. In addition to

\footnotetext{
${ }^{9}$ I will comment in $\S 9$ on how the analysis is modified if a different error model is adopted.
} 
these storage errors that afflict the "resting" qubits, there will also be errors that are introduced by the quantum gates themselves. For each type of gate, the probability of error each time the gate is implemented is denoted $\epsilon_{\text {gate }}$ (with independent values assigned to gates of each type). If the gate acts on more than one qubit (XOR or Toffoli), correlated errors may arise. In our analysis, we make the pessimistic assumption that an error in a multi-qubit gate always damages all of the qubits on which the gate acts; e.g., a faulty XOR gate introduces errors in both the source qubit and the target qubit. This assumption (among others) is made only to keep the analysis tractable. Under more realistic assumptions, we would surely find that somewhat higher error rates could be tolerated.

We can analyze the efficacy of concatenated coding by constructing a set of concatenation flow equations, that describe how our error model evolves as we proceed from one level of concatenation to the next. For example, suppose we want to perform an XOR gate followed by an error recovery step on qubits encoded using the concatenated Steane code with $L$ levels of concatenation (block size $7^{L}$ ). The gate implementation and recovery can be described in terms of operations that act on subblocks of size $7^{L-1}$. Thus, we can derive an expression for the probability of error $\epsilon^{(L)}$ for a gate acting on the full block in terms of the probability of error for gates acting on the subblocks. This expression is one of the flow equations. In principle, we can solve the flow equations to find the error probabilities "at level $L$ " in terms of the parameters of the error model for the elementary qubits. We then study how the error probabilities behave as $L$ becomes large. If all block error probabilities approach zero for $L$ large, then the elementary error probabilities are "below the threshold." Since our elementary error model may have many parameters, the threshold is really a codimension one surface in a high-dimension space.

The flow equations are intricate; for a detailed description see Gottesman (1997b), Gottesman et al. (1996), or Zalka (1996). But to crudely illustrate the idea, let us suppose that there are no storage errors, and that the only gates that cause errors are the XOR gates, with a probability of error per gate given by $\epsilon_{\mathrm{XOR}}$. Suppose that we wish to implement a network of XOR gates that act on encoded blocks. Let us estimate how small $\epsilon_{\mathrm{XOR}}$ must be for the error rate achieved by gates acting on encoded data to be smaller than the error rate for the elementary XOR gates.

Using Shor's ancilla state, 12 XOR gates are needed to compute either the bit flip or phase flip syndrome 10 so the probability of error for each syndrome measurement is

$$
\epsilon_{\text {syndrome }} \sim 12 \epsilon_{\mathrm{XOR}} .
$$

To ensure that the syndrome is reliable, it is measured repeatedly until the same result is obtained twice in a row. ${ }^{17}$ From some simple combinatorics, one sees that the probability that two independent errors occur before the syndrome measurement is successfully completed is $5 \epsilon_{\text {syndrome }}^{2}$. If we assume that both errors damage the data, then two errors will result in an uncorrectable error in the block. Since we need to measure both the bit-flip syndrome and the phase-flip syndrome, we see that the probability of failure each time we perform recovery is

$$
\epsilon_{\text {failure }} \sim 2 \cdot 5 \cdot\left(12 \epsilon_{\mathrm{XOR}}\right)^{2}=1440 \epsilon_{\mathrm{XOR}}^{2} .
$$

In the implementation of a network of XOR gates, we will perform recovery on each block after it has been acted on by $N$ gates, where $N$ is a number that will be chosen to optimize the error per gate. Each transversal XOR gate acting on a block is performed by implementing 7 elementary XOR gates. When we perform $N$ gates followed by recovery on a given block, the probability per gate that two errors accumulate in the block is no worse than

$$
\begin{aligned}
\epsilon_{\text {fail }} & \sim \frac{1}{N}\left(\frac{1}{2} 7 N \cdot 6 N \cdot \epsilon_{\mathrm{XOR}}^{2}+7 N \cdot \epsilon_{\mathrm{XOR}} \cdot 2 \cdot 2 \cdot \epsilon_{\mathrm{Syndrome}}+1440 \cdot \epsilon_{\mathrm{XOR}}^{2}\right) \\
& =\frac{1}{N}\left(21 N^{2}+336 N+1440\right) \epsilon_{\mathrm{XOR}}^{2}
\end{aligned}
$$

\footnotetext{
${ }^{10}$ This number would be reduced to 7 if Steane's ancilla state were used; however, since the preparation of the Steane state is more complex, the ancilla would be more prone to error.

${ }^{11}$ This is not really the optimal procedure, but we will adopt it here for simplicity.
} 
(There might be two errors introduced by gates, one from a gate and one from the recovery step, or two during recovery: ${ }_{2}^{12}$ we pessimistically assume that all the errors damage the data in the block, and that recovery always fails if two errors occur within a data block.) The minimum of Eq. 27 occurs if we choose $N=8$, and we then obtain $\epsilon_{\text {fail }}=684 \epsilon_{\mathrm{XOR}}^{2}$. So coding pays off if $684 \epsilon_{\mathrm{XOR}}^{2}<\epsilon_{\mathrm{XOR}}$, or $\epsilon_{\mathrm{XOR}}<(684)^{-1} \sim 1.5 \cdot 10^{-3}$. This is our "threshold estimate."

Even if all errors were due to XOR gates, this estimate would be inadequate for a number of reasons. Most importantly, we have assumed that the ancilla states that are used in syndrome measurement are error free, while in fact XOR gates are used in the preparation and verification of the Shor state. Furthermore, when XOR gates act on the subblocks of a concatenated code, it is not always possible to implement the optimal number of gates before recovery. And of course a more complete analysis would incorporate the storage errors, and would track the coupled flow of storage and gate errors as a level of concatenation is added.

Indeed, when we perform recovery at level- $L$, the Shor states must be constructed from blocks that are encoded at level- $(L-1)$. An important part of the analysis is to monitor carefully how noisy these ancilla states are, in order to determine how likely it is for recovery to fail at level- $L$. The data blocks need to wait while the ancilla states are prepared; meanwhile storage errors are accumulating in the data. Because the waiting period does not scale simply with the level $L$, the flow equations are not entirely self-similar - there is some explicit "time-dependence" (that is, $L$-dependence) in the flow. It is still roughly true, though, that the threshold is established by demanding that the fault-tolerant procedure with a single level of concatenation really improves the reliability of the gates, as in the above example.

Though the flow equations are too complicated to solve exactly, we can obtain approximate solutions by making conservative assumptions. Crudely speaking, the conclusions are as follows (Gottesman et al. 1996; Gottesman 1997b): If storage errors are negligible, then the threshold rate for gate errors is about $10^{-4}$. If storage errors dominate, then the threshold error rate is about $10^{-5}$ per time step (where the unit of time is the time required to implement one gate). The elementary Toffoli gates are not required to be as accurate as the one and two-body gates - an Toffoli gate error rate of $10^{-3}$ is acceptable, if the other error rates are sufficiently small. (This finding is welcome, since Tofolli gates are more difficult to implement, and are likely to be less accurate in practice.)

We should also ask how large a block size is needed to ensure a certain specified accuracy. Roughly speaking, if the threshold gate error rate is $\epsilon_{0}$ and the actual elementary gate error rate is $\epsilon<\epsilon_{0}$, then concatenating the code $L$ times will reduce the error rate to

$$
\epsilon^{(L)} \sim \epsilon_{0}\left(\frac{\epsilon}{\epsilon_{0}}\right)^{2^{L}} ;
$$

thus, to be reasonably confident that we can complete a computation with $T$ gates without making an error we must choose the block size $7^{L}$ to be

$$
\text { size } \sim\left[\frac{\log \epsilon_{0} T}{\log \epsilon_{0} / \epsilon}\right]^{\log _{2} 7}
$$

If the code that is concatenated has block size $n$ and can correct $t+1$ errors, the power $\log _{2} 7 \sim 2.8$ in Eq. 29 is replaced by $\log n / \log (t+1)$; this power approaches 2 for the family of codes considered by Shor, but could in principle approach 1 for "good" codes.

When the error rates are below the accuracy threshold, it is also possible to maintain an unknown quantum state for an indefinitely long time. However, as we have already noted in $\S$, if the probability of a storage error per computational time step is $\epsilon$, then the initial encoding of the state can be performed with a fidelity no better than $F=1-O(\epsilon)$. With concatenated coding, we can store

\footnotetext{
${ }^{12}$ Of the two factors of 2 in the second term of Eq. 27, one arises because we measure both the bit-flip and phaseflip syndromes, the other because the error can occur during either the first or second repetition of the syndrome measurement.
} 


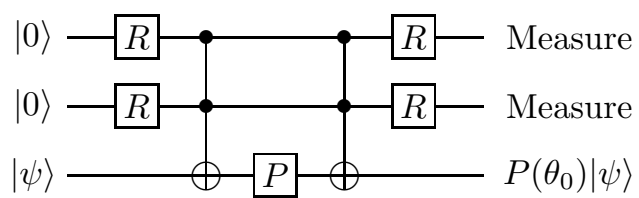

Figure 14: A rotation circuit. If the measurement outcome is $(00)$, then the rotation $P\left(\theta_{0}\right)$ is applied to the data qubit, where $\cos \left(\theta_{0}\right)=3 / 5$.

unknown quantum information with reasonably good fidelity for an indefinitely long time, but we cannot achieve arbitrarily good fidelity.

The assumptions that underlie these conclusions will be reviewed in $\S 9$.

\section{Fault-tolerant factorization}

To get an idea what the scaling law Eq. 29 might mean in practice, consider using concatenated coding to implement Shor's (1994) quantum factoring algorithm. The algorithm has two parts. To factor the number $N$, we must first evaluate the modular exponential function to prepare a state of the form

$$
\sum_{x}|x\rangle_{\text {input }} \otimes\left|a^{x}(\bmod N)\right\rangle_{\text {output }},
$$

(where $a<N$ is relatively prime to $N$ ), and then perform a Fourier transform acting on the input register. Finally, we measure the input register, and do some classical post-processing to find a candidate factor of $N$. The preparation of the state Eq. 30 (using the fault tolerant gates listed in $\S$ 司) is described in Beckman et al. (1996) and Vedral et al. (1996). The Fourier transform requires more comment. As shown by Griffiths and Niu (1996), the Fourier transform can be evaluated with single-qubit gates (but with the type of gate conditioned on the outcome of previous measurements). In particular, we will need to be able to perform phase rotation gates of the form

$$
P(\theta)=\left(\begin{array}{cc}
1 & 0 \\
0 & e^{i \theta}
\end{array}\right)
$$

It suffices to have a fault-tolerant procedure for implementing $P\left(\theta_{0}\right)$ for a particular $\theta_{0}$ that is an irrational multiple of $2 \pi$, since then applying $P\left(\theta_{0}\right)$ repeatedly allows us to come arbitrarily close to $P(\theta)$ for any value of $\theta$.

To construct $P\left(\theta_{0}\right)$ we need to use ancilla bits and apply the Toffoli gate. One circuit that works is shown in Fig. 14. When the two ancilla bits are measured, the probability is $\frac{5}{8}$ that the outcome is

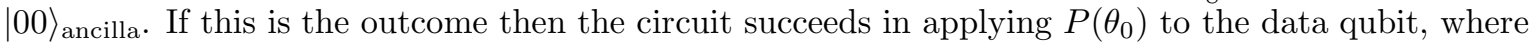
$e^{i \theta_{0}}=(1+3 i) /(3+i)$, or $\cos \left(\theta_{0}\right)=\frac{3}{5}$. For any other outcome $(|01\rangle,|10\rangle$, or $|11\rangle$, each occurring with probability $\left.\frac{1}{8}\right)$, the circuit fails. But we can repair the damage to the qubit by applying the $Z$-gate, and then make further attempts to apply $P\left(\theta_{0}\right)$ until we finally succeed.

An alternate method is to assemble "off-line" a library of "angle qubits" of the form

$$
|\theta\rangle=\frac{1}{\sqrt{2}}\left(|0\rangle+e^{i \theta}|1\rangle\right)
$$

To apply $P(\theta)$ to a data qubit, we perform a controlled-NOT with the data qubit as the control and the library qubit $|\theta\rangle$ as the target, as in Fig. 15. We then measure the library qubit. With probability $\frac{1}{2}$, the outcome of the measurement will be $|0\rangle$, and if so then we have successfully applied $P(\theta)$ to 


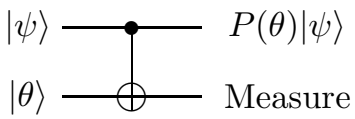

Figure 15: A rotation circuit that employs an ancilla qubit withdrawn from the angle library. If the measurement outcome is 0 , then $P(\theta)$ is applied to the data qubit.

the data qubit. But if the outcome is $|1\rangle$, then we have applied $P(-\theta)$ instead. In the event of failure, we make another attempt, but this time we try to apply $P(2 \theta)$, to compensate for the error in the previous step. The probability of failing $n$ times in a row is only $2^{-n}$.

Now let us attempt to factor a $K$-bit number using the factoring circuit of Beckman et al. (1996), employing the first method described above to perform the single-qubit rotations required in the Fourier transform. To perform the Fourier transform (on a register with $2 K$ qubits), $2 K$ single-qubit phase rotations are needed. The Fourier transform need not be evaluated with perfect precision; for the factoring algorithm to have a reasonable chance of succeeding, it is sufficient for each rotation to have a precision of order $K^{-1}$. We can achieve this accuracy by composing $P\left(\theta_{0}\right)$ of order $K$ times. Hence the Fourier transform requires of order $K^{2}$ Toffoli gates, and we conclude that for large $K$, the complexity of the algorithm is dominated by the evaluation of the modular exponential function, which requires $38 K^{3}$ Toffoli gates according to Beckman et al. (1996).13

With the best known classical algorithms and the fastest existing machines, it takes of order a few months to factor a 130 digit $(K \sim 430$ bit) number (Lenstra et al. 1996). Let's ask what resources a quantum computer would need to perform this task. The machine would need to be able to store $5 K \sim 2150$ encoded qubits, and to perform of order $3 \cdot 10^{9}$ Toffoli gates. For there to be a reasonable probability of performing the computation without an error, we would like the probability of error per Toffoli gate to be less than about $10^{-9}$, and the probability of a storage error per gate execution time to be less than about $10^{-12}$. According to the concatenation flow equations, these error rates can be achieved for the encoded data, if the error rates at the level of individual qubits are $\epsilon_{\text {store }} \sim \epsilon_{\text {gate }} \sim 10^{-6}$, and if 3 levels of concatenation are used, so that the size of the block encoding each qubit is $7^{3}=343$. Allowing for the additional ancilla qubits needed to implement gates and (parallelized) error correction, the total number of qubits required in the machine would be of order $10^{6}$.

With the error rates of order $10^{-6}$ for the individual qubits, concatenating the 7 -qubit code may be the most effective fault-tolerant procedure. For error rates about an order of magnitude smaller, we might do better with a more complex (unconcatenated) code that can correct several errors in a single block (Shor 1996). At still lower error rates it is possible to use codes that make more efficient use of storage space by encoding many qubits in a single block (Gottesman 1997a). In fact, when the error rate for individual qubits is very low, it becomes possible in principle to find a good code for fault-tolerant computation such that the ratio of encoded qubits to total qubits approaches one.

\section{Plumbing quantum leaks}

I would now like to examine a little more closely one of the assumptions made in the above analysis. We have ignored the possibility of leakage. In our model of a quantum computer, each of our qubits lives in a two-dimensional Hilbert space. We assumed that, when an error occurs, this qubit either becomes entangled with the environment, or rotates in the two-dimensional space in an unpredictable way. But there is another possible type of error, in which the qubit leaks out of the two-dimensional

\footnotetext{
${ }^{13}$ The algorithm described by Beckman et al. actually requires $46 K^{3}$ Toffoli gates, but this can be reduced to $38 K^{3}$ using an improved comparison algortihm suggested by Richard Hughes (1997).
} 


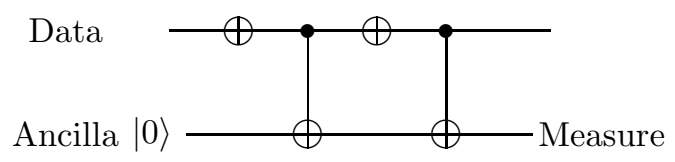

Figure 16: A quantum leak detection circuit. The outcome of the measurement is 0 if leakage has occurred, 1 otherwise.

space into a larger space (Plenio \& Knight 1996). For example, in an ion trap computer we might store quantum information in a two-dimensional space spanned by the ground state of the ion and a particular long-lived metastable state (Cirac \& Zoller 1995). But during the operation of the device, the ion might make an unexpected transition to another state. If that state decays quickly to the ground state, then the error can be detected and corrected using the now standard methods of faulttolerant quantum error correction. But if the ion remains hung up in the wrong state for a long time, those methods will fail.

One strategy for dealing with the leakage problem would be to identify the levels that are the most likely candidates for leakage, and to repeatedly pump these levels back to the ground state, but this strategy might be unwieldy if there are many candidate levels. A more elegant solution is to detect the leakage, but without trying to diagnose exactly what happened to the leaked qubit. For example, in an ion trap, we might design a controlled-NOT gate that acts trivially if the control bit does not reside in the two-dimensional space where the qubit belongs. Then we can carry out the gate sequence shown in Fig. 16. Nothing happens if the data qubit has leaked, but in the event that no leakage has occurred, the ancilla bit will flip. We then measure the ancilla bit. The measurement result 1 projects the qubit to the right Hilbert space, but the result 0 projects the qubit to a leaked state.

If leakage has occurred, the qubit is damaged and must be discarded.14 We replace it with a fresh qubit in a standard state, say the state $|0\rangle$. (If concatenated coding is used, leakage detection need be implemented only at the lowest coding level.) Now we can perform conventional syndrome measurement, which will project the qubit onto a state such that the error can be reversed by a simple unitary transformation. In fact, since we know before the syndrome measurement that the damaged qubit is in a particular position within the code block, we can apply a streamlined version of error correction designed to diagnose and reverse the error at that known position (Grassl et al. 1996).

\section{Dream machine}

Let's recall some of the important assumptions that we made in our estimate of the accuracy threshold:

- Random errors. We have assumed that the errors have no systematic component. ${ }^{5}$ This assumption enables us to add probabilities, rather than amplitudes, to estimate how the probability of error accumulates over time. Systematic errors would accumulate much more rapidly, and therefore the error rate that can be tolerated would be much lower. Roughly, if the accuracy threshold is $\epsilon_{0}$ for random errors, it would be of order $\left(\epsilon_{0}\right)^{2}$ for maximally conspiratorial systematic errors. My attitude is that (1) even if our hardware is susceptible to making errors with systematic phases, these will tend to cancel out in the course of a reasonably long computation (Obenland \& Despain 1996ab; Miquel et al. 1997), and (2) since systematic errors can in principle be understood and eliminated, from a fundamental point of view it is more relevant to know the limitations on the performance of the machine that are imposed by the random errors.

\footnotetext{
${ }^{14}$ Of course, we can recycle it later after it returns to the ground state.

${ }^{15}$ Knill et al. $(1996,1997)$ have demonstrated the existence of an accuracy threshold for much more general error models.
} 
- Uncorrelated errors. We have assumed that the errors are both spatially and temporally uncorrelated with one another. Thus when we say that the probability of error per qubit is $\epsilon \sim 10^{-5}$, we actually mean that, given two specified qubits, the probability that errors afflict both is $\epsilon^{2} \sim 10^{-10}$. This is a strong assumption, and an important one, since our coding schemes will fail if several errors occur in the same block of the code. Future quantum engineers will face the challenge of ensuring that this assumption is justified reasonably well in the devices that they design.

- Maximal parallelism. We have assumed that many quantum gates can be executed in parallel in a single time step. This assumption enables us to perform error recovery in all of our code blocks at once, and so is critical for controlling qubit storage errors. (Otherwise, if we added a level of concatenation to the code, each individual resting qubits would have to wait longer for us to get around to perform recovery, and would be more likely to fail.) If we ignore storage errors, then parallel operation is not essential in the analysis of the accuracy threshold, but it would certainly be desirable to speed up the computation.

- Error rate independent of number of qubits. We have assumed that the error rates do not depend on how many qubits are stored in our device. Implicitly, this is an assumption about the nature of the hardware. For example, it would not be a reasonable assumption if all of the qubits were stored in a single ion trap, and all shared the same phonon bus (Cirac \& Zoller 1995).

- Gates can act on any pair of qubits. We have assumed that our machine is equipped with a set of fundamental gates that can be applied to any pair of stored qubits (or triplet of qubits, in the case of the Toffoli gate), irrespective of their proximity. In practice, there is likely to be a cost, both in processing time and error rate, of shuttling the qubits around so that a gate can act effectively on a particular pair. We leave it to the machine designer to choose an architecture that minimizes this cost.

- Fresh ancilla qubits. We have assumed that our computer has access to an adequate supply of fresh ancillary qubits. The ancilla qubits are used both to implement (Toffoli) gates and to perform error recovery. As the effects of random errors accumulate, entropy is generated, and the error recovery process flushes the entropy from the computing device into the ancilla registers. In principle, the computation can proceed indefinitely as long as fresh ancilla qubits are provided, but in practice we will want to clear the ancilla and reuse it. Erasing the ancilla will necessarily dissipate power and generate heat; thus cooling of the device will be required.

- No leakage errors. Leakage errors have been ignored. But as noted in $\S \S$, including them would not have a big effect on the conclusions.

Our assumptions have been sufficiently realistic that we are justified in concluding that a quantum computer with about a million qubits and an error rate per gate of about one in a million would be a powerful and valuable device (assuming a reasonable processing speed). From the perspective of the current state of the technology (Monroe et 1al. 1995; Turchette et al. 1995; Cory et al. 1996; Gershenfeld \& Chuang 1997), these numbers seem daunting. But in fact a machine that meets far less demanding specifications may still be very useful (Preskill 1997). First of all, quantum computers can do other things besides factoring, and some of these other tasks (in particular quantum simulation Lloyd 1996) might be accomplished with a less reliable or smaller device. Furthermore, our estimate of the accuracy threshold might be too conservative for a number of reasons. For example, the estimate was obtained under the assumption that phase and amplitude errors in the qubits are equally likely. With a more realistic error model better representing the error probabilities in an actual device, the error correction scheme could be better tailored to the error model, and a higher error rate could be tolerated. Also, even under the assumptions stated, the fault-tolerant scheme has not been definitively analyzed; with a more refined analysis, one can expect to find a somewhat higher accuracy threshold, 
perhaps considerably higher. Substantial improvements might also be attained by modifying the faulttolerant scheme, either by finding a more efficient way to implement a universal set of fault-tolerant gates, or by finding a more efficient means of carrying out the measurement of the error syndrome. With various improvements, I would not be surprised to find that a quantum computer could work effectively with a probability of error per gate, say, of order $10^{-4}$. (That is $10^{-4}$ may turn out to be comfortably below the accuracy threshold. In fact, estimates of the accuracy threshold that are more optimistic than mine have been put forward by Zalka (1996).)

Anyway, if we accept these estimates at face value, we now have a rough target to aim at: $10^{6}$ qubits with a $10^{-6}$ error rate. That sounds pretty tough. But of course it might have been worse. If we had found that we need an error rate of order, say, $10^{-20}$, then the future prospects for quantum computing would be dim indeed. An error rate of $10^{-6}$ is surely ambitious, but not, perhaps, beyond the scope of what might be achievable in the future. In any case, we now have a fair notion of how good the performance of a useful quantum computer will need to be. And that in itself represents enormous progress over just a year ago.

This work has been supported in part by the Department of Energy under Grant No. DE-FG03-92ER40701, and by DARPA under Grant No. DAAH04-96-1-0386 administered by the Army Research Office. I am grateful to David DiVincenzo and Wojciech Zurek for organizing this stimulating meeting, and I thank Andrew Steane and Christof Zalka for helpful comments on the manuscript. I also wish to thank my collaborators David Beckman, Jarah Evslin, Sham Kakade, and especially Daniel Gottesman for many productive discussions about fault-tolerant quantum computation.

\section{References}

Aharonov, D. \& Ben-Or, M. 1996a Fault tolerant quantum computation with constant error. (Online preprint quant-ph/9611025.)

Beckman, D., Chari, A., Devabhaktuni, S. \& Preskill, J. 1996 Efficient networks for quantum factoring. Phys. Rev. A 54, 1034.

Bennett, C., DiVincenzo, D., Smolin, J. \& Wootters, W. 1996 Mixed state entanglement and quantum error correction. Phys. Rev. A 54, 3824.

Calderbank, A. R. \& Shor, P. W. 1996 Good quantum error-correcting codes exist. Phys. Rev. A $\mathbf{5 4}, 1098$.

Calderbank, A. R., Rains, E. M., Shor, P. W. \& Sloane, N. J. A. 1996 Quantum error correction via codes over GF(4). (Online preprint quant-ph/9608006.)

Calderbank, A. R., Rains, E. M., Shor, P. W. \& Sloane, N. J. A. 1997 Quantum error correction and orthogonal geometry. Phys. Rev. Lett. 78, 405.

Cirac, J. I. \& Zoller, P. 1995 Quantum computations with cold trapped ions. Phys. Rev. Lett. 74, 4091.

Cory, D. G., Fahmy, A. F. \& Havel, T. F. 1996 Nuclear magnetic resonance spectroscopy: an experimentally accessible paradigm for quantum computing. In Proceedings of the 4th Workshop on Physics and Computation, Boston: New England Complex Systems Institute.

Dieks, D. 1982 Communication by electron-paramagnetic-resonance devices. Phys. Lett. A 92, 271.

DiVincenzo, D. \& Shor, P. 1996 Fault-tolerant error correction with efficient quantum codes. Phys. Rev. Lett. 77, 3260 .

Gershenfeld, N. \& Chuang, I. 1997 Bulk spin resonance quantum computation. Science 275, 350. 
Griffiths, R. B. \& Niu, C. 1996 Semiclassical Fourier transform for quantum computation. Phys. Rev. Lett. 76, 3228.

Gottesman, D. 1996 Class of quantum error-correcting codes saturating the quantum Hamming bound. Phys. Rev. A 54, 1862.

Gottesman, D. 1997a A theory of fault-tolerant quantum computation. (Online preprint quant$\mathrm{ph} / 9702029$.)

Gottesman, D. 1997b Stabilizer codes and quantum error correction. Ph.D. thesis, California Institute of Technology.

Gottesman, D., Evslin, J. Kakade, S. \& Preskill, J. 1996, to be published.

Grassl, M., Beth, Th. \& Pellizzari, T. 1996 Codes for the quantum erasure channel. (Online preprint quant-ph/9610042.)

Hughes, R. 1997 (private communication).

Kitaev, A. Yu. 1996a Quantum error correction with imperfect gates, preprint.

Kitaev, A. Yu. 1996b Quantum computing: algorithms and error correction, preprint (in Russian).

Knill, E. \& Laflamme, R. 1996 Concatenated quantum codes. (Online preprint quant-ph/9608012.)

Knill, E. \& Laflamme, R. 1997. A theory of quantum error-correcting codes. Phys. Rev. A 55, 900.

Knill, E., Laflamme, R. \& Zurek, W. 1996 Accuracy threshold for quantum computation. (Online preprint quant-ph/9610011.)

Knill, E., Laflamme, R. \& Zurek, W. 1997 Resilient quantum computation: error models and thresholds. (Online preprint quant-ph/9702058.)

Laflamme, R., Miquel, C., Paz, J. P., \& Zurek, W. 1996 Perfect quantum error correction code. Phys. Rev. Lett. 77, 198.

Landauer, R. 1995 Is quantum mechanics useful? Phil. Tran. R. Soc. Lond. 353, 367.

Landauer, R. 1996 The physical nature of information. Phys. Lett. A 217, 188.

Landauer, R. 1997 Is quantum mechanically coherent computation useful? In Proc. Drexel-4 Symposium on Quantum Nonintegrability-Quantum-Classical Correspondence, Philadelphia, PA, 8 September 1994 (ed. D. H. Feng and B.-L. Hu), Boston: International Press.

Lenstra, A. K., Cowie, J., Elkenbracht-Huizing, M., Furmanski, W., Montgomery, P. L., Weber, D. \& Zayer, J. 1996 RSA factoring-by-web: the world-wide status. (Online document http: //www.npac.syr.edu/factoring/status.html.)

Lloyd, S. 1996 Universal quantum simulators. Science 273, 1073.

Lloyd, S. 1997 The capacity of a noisy quantum channel. Phys. Rev. A 55, 1613.

MacWilliams, F. J. \& Sloane, N. J. A. 1977 The Theory of Error-Correcting Codes. New York: North-Holland Publishing Company.

Miquel, C. Paz, J. P. \& Zurek, W. H. 1997 Quantum computation with phase drift errors. (Online preprint quant-ph/9704003.)

Monroe, C., Meekhof, D. M., King, B. E., Itano, W. M. \& Wineland, D. J. 1995 Demonstration of a fundamental quantum logic gate. Phys. Rev. Lett. 75, 4714. 
Obenland, K. \& Despain, A. M. 1996a Simulation of factoring on a quantum computer architecture. In Proceedings of the 4th Workshop on Physics and Computation, Boston, November 22-24, 1996, Boston: New England Complex Systems Institute.

Obenland, K. \& Despain, A. M. 1996b Impact of errors on a quantum computer architecture. (Online preprint http://www.isi.eu/acal/quantum/quantum_op_errors.ps.)

Plenio, M. B. \& Knight, P. L. 1996 Decoherence limits to quantum computation using trapped ions. (Online preprint quant-ph/9610015.)

Preskill, J. 1997 Quantum computing: pro and con. (Online preprint quant-ph/9705032.)

Shor, P. 1994 Algorithms for quantum computation: discrete logarithms and factoring. In Proceedings of the 35th Annual Symposium on Fundamentals of Computer Science. Los Alamitos, CA: IEEE Press, pp. 124-134.

Shor, P. 1995 Scheme for reducing decoherence in quantum memory. Phys. Rev. A 52, 2493.

Shor, P. 1996 Fault-tolerant quantum computation. In Proceedings of the Symposium on the Foundations of Computer Science. Los Alamitos, CA: IEEE Press (Online preprint quant-ph/9605011).

Shor, P. 1997, these proceedings.

Shor, P. \& and Smolin, J. 1996 Quantum error-correcting codes need not completely reveal the error syndrome. (Online preprint quant-ph/9604006.)

Steane, A. M. 1996a Error correcting codes in quantum theory. Phys. Rev. Lett. 77, 793.

Steane, A. M. 1996b Multiple particle interference and quantum error correction. Proc. Roy. Soc. Lond. A 452, 2551.

Steane, A. M. 1997 Active stabilization, quantum computation and quantum state synthesis. Phys. Rev. Lett. 78, 2252.

Turchette, Q. A., Hood, C. J., Lange, W., Mabuchi, H. \& Kimble, H. J. 1995 Measurement of conditional phase shifts for quantum logic. Phys. Rev. Lett. 75, 4710 (1995).

Vedral, V., Barenco, A. \& Ekert, A. 1996 Quantum networks for elementary arithmetic operations. Phys. Rev. A 54, 139

Unruh, W. G. 1995 Maintaining coherence in quantum computers. Phys. Rev. A 51, 992.

Wootters, W. K. \& Zurek, W. H. 1982 A single quantum cannot be cloned. Nature 299, 802.

Zalka, C. 1996 Threshold estimate for fault tolerant quantum computing. (Online preprint quant$\mathrm{ph} / 9612028$.) 